\title{
Kinderonkologie: Erhaltungspflege von Broviac- und Port-Kathetern
}

Max Scheler, Arne Simon

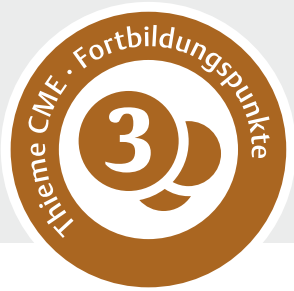

\begin{abstract}
Die meisten Kinder mit Krebs erhalten zu Beginn der Chemotherapie einen zentralen Venenkatheter. Die Prävention von Blutstrominfektionen, die von solchen Gefäßkathetern ausgehen, ist ein wichtiges Merkmal guter klinischer Praxis. Dieser Beitrag diskutiert verschiedene Aspekte von Präventionsbündeln in diesem speziellen klinischen Kontext.
\end{abstract}

\section{Einleitung}

Bei kinderonkologischen Patienten wird meist schon zu Beginn der zytostatischen Behandlung [39] ein dauerhafter zentraler Gefäßkatheter (central versus access device; CVAD) implantiert [84, 87] - entweder als getunnelter Broviac-/Hickman-Katheter oder voll implantierter Port-Katheter. Diese CVADs sind ein unverzichtbarer Bestandteil des gesamten Behandlungskonzepts. Sie dienen dazu, zytotoxische Medikamente zu verabreichen, bei denen ein hohes Risiko für eine Gewebsnekrose besteht, wenn sie versehentlich ins Gewebe gelangen (Paravasat). Außerdem lassen sich über den CVADTherapiebegleitinfusionen geben, in denen Elektrolyte wie Kalium, Kalzium, oder Magnesium in Konzentrationen enthalten sind, die bei einer periphervenösen Verabreichung nicht toleriert werden (Phlebitis). Des Weiteren erhalten die Patienten über den CVAD Schmerzmedikamente (z. B. Morphin), parenterale Ernährung, Antiinfektiva und Blutprodukte (Transfusionen). Die allermeisten Blutentnahmen (inklusive der Blutkulturen bei Infektionsverdacht) [30] erfolgen bei kinderonkologischen Patienten über den CVAD. Die Schmerzfreiheit der Blutentnahme ist ein wesentlicher, patientenzentrierter Vorteil von CVADs.

Bei Kindern mit Krebserkrankungen machen Blutstrominfektionen (BSI), die durch bakterielle Erreger ausgelöst werden, in etwa die Hälfte aller nosokomialen Infektionen mit Erregernachweis aus [2 - 7, 53, 54, $68,73,83,85]$. Durch die hohe CVAD-Anwendungsrate - über $85 \%$ der Patienten haben einen CVAD - , ist ein erheblicher Anteil aller BSI bei kinderonkologischen Patienten mit einem Gefäßkatheter assoziiert.

\section{Merke}

Nur ein Teil der Blutstrominfektionen geht tatsächlich vom Gefäßkatheter aus (Catheter-related BSI= CR-BSI, Katheter-assoziierte BSI=CA-BSI). Diese unerwünschten Komplikationen lassen sich zum Teil durch gezielte Präventionsmaßnahmen verhindern. $[37,86]$ (s. Fallbeispiel 1).

\section{FALLBEISPIEL 1}

Bei einem Jugendlichen mit Ewing-Sarkom wird erstmals eine Woche nach Ende des letzten Chemotherapieblocks (Vincristin, Ifosfamid, Doxorubicin, Etoposid) bei einer Granulozytenzahl von $0,4 \times 10^{9} / /$ eine Rötung der Broviac-Eintrittstelle ohne Schmerzen, Schwellung oder Exsudat beobachtet. Es erfolgt ein Abstrich für die Mikrobiologie (CoNS: Koagulase-negative Staphylokokken, Methicillin-resistent) und eine lokale Behandlung mit Octenisept ${ }^{\circledR}$ und medizinischem Honig $\left(\right.$ Medihoney $^{\mathrm{TM}}$ ) eingeleitet. Die Eltern führen den Verbandswechsel am Broviac selbst täglich durch, die Rötung geht langsam zurück. Wahrscheinlich ist diese Rötung durch die Chemotherapie verursacht worden. Die an der Eintrittsstelle nachgewiesenen CoNS sind nur besiedelnde Bakterien.

Die intensive Chemotherapie (v.a. hoch dosiertes Methotrexat, Anthrazykline, hoch dosiertes Cytarabin, hoch dosiertes Etoposid) und ggf. auch die Strahlentherapie verursachen neben einer Granulozytopenie (Granulozyten<0,5 × 109/I) auch eine Schädigung der mukosalen Barriere im Oropharynx und in den tieferen Abschnitten des Gastrointestinaltrakts (Mukositis; mucosal barrier injury) $[20,101]$.

\footnotetext{
Merke

Sowohl die Granulozytopenie als auch die Mukositis erhöhen das Risiko einer Translokation von Bakterien von den Schleimhäuten des Gastrointestinaltrakts ins Blut [77]. Dies gilt z. B. für vergrünende Streptokokken, Enterobakterien (E. coli, Klebsiella spp., Enterobacter spp.) und für Enterokokken (E. faecium und E. faecalis) [91] (s. Fallbeispiel 2).
} 


\section{FALLBEISPIEL 2}

Eine 12-jährige Patientin mit akuter myeloischer Leukämie (AML) wird 6 Tage nach der zweiten Induktionstherapie (hoch dosiertes Cytarabin, Mitoxantron) in deutlich reduziertem Allgemeinzustand mit Fieber (oral gemessen $39^{\circ} \mathrm{C}$ ), verlängerter Rekapillarisierungszeit, Tachykardie und Tachypnoe stationär aufgenommen. Die Patientin hat zuhause nur wenig getrunken (starke Mundschmerzen, im Verlauf Morphin, parenterale Ernährung).

Der Hb-Wert beträgt $6,8 \mathrm{~g} / \mathrm{dl}$, die Thrombozyten sind bei $14 \times 10^{9} / \mathrm{I}$ und die Leukozyten bei $0,2 \times 10^{9} / \mathrm{I}$. Das C-reaktive Protein im Serum liegt initial bei $65 \mathrm{mg} / \mathrm{l}$, steigt jedoch am zweiten Tag auf $240 \mathrm{mg} / \mathrm{l}$ an. Bei Aufnahme erhält die Patientin Piperacillin-Tazobactam und Teicoplanin, außerdem Volumen (allein $40 \mathrm{ml} / \mathrm{kg}$ in der ersten Stunde), Erythrozytenkonzentrat und Thrombozytenkonzentrat. In den beiden Blutkultursets (aerob und anaerob) aus dem Broviac-Katheter wachsen vergrünende Streptokokken, die in vitro sensibel gegen Piperacillin und Teicoplanin sind.

Die Patientin muss am ersten Tag nach Aufnahme wegen Hypotonie, Oligurie und zunehmendem Sauerstoffbedarf auf die Intensivstation verlegt und dort vorübergehend mit Noradrenalin behandelt werden. Eine Intubation und Beatmung lässt sich durch den Einsatz einer nicht invasiven Atemunterstützung über eine Maske vermeiden.

Die Kontrollblutkulturen $24 \mathrm{~h}$ nach Aufnahme zeigen kein Wachstum. Der Broviac-Katheter wird wechselseitig (2 Lumina) für 2 h mit Ethanol $80 \%$ geblockt. Nach 3 Tagen kann die Patientin wieder auf die kinderonkologische Station verlegt werden. Die antibiotische Therapie wird insgesamt 10 Tage fortgeführt. Nach Auffassung der behandelnden Onkologen liegt hier eine Translokationsbakteriämie bei Chemotherapieinduzierter Granulozytopenie und Mukositis vor.

\section{Merke}

Bakterielle BSI können in Phasen der ausgeprägten Immunsuppression das Leben der Patienten akut gefährden [67]. Sie erfordern eine sofortige stationäre Aufnahme und intravenöse Antibiotikatherapie $[8,12,30,59]$, wodurch die Lebensqualität der Patienten und ihrer Familien (im Vergleich zu Kindern ohne eine solche Infektion) signifikant beeinträchtigt wird [24, 31].

Des Weiteren erhöhen BSI die Behandlungskosten: bei sehr konservativer Kalkulation um mindestens $4400 €$ pro Ereignis [19,94], nach einer aktuellen US-amerikanischen Studie um 70000 US\$ (Stand 2011; um 21 Tage verlängerte Liegedauer) [106].

Die empirische (kalkulierte) und die gezielte Therapie (bei positiver Blutkultur) von BSI steigern den Selektionsdruck auf bakterielle Erreger mit speziellen Resistenzen und Multiresistenzen in kinderonkologischen Abteilungen [11, 30, 38, 63]. Mit dem CVAD assoziierte oder von diesem ausgehende BSI durch Methicillin-resistente, Koagulase-negative Staphylokokken sind ein wesentlicher Antrieb des Einsatzes von Glykopeptiden bei kinderonkologischen Patienten [88].

\section{Merke}

Die Antibiotikatherapie erhöht beim individuellen Patienten das Risiko einer C.-difficile-assoziierten Enterokolitis [78, 104]. Außerdem können BSI und deren Therapie die Chemotherapie relevant verzögern, was sich möglicherweise ungünstig auf die Gesamtprognose auswirkt.

Vor diesem Hintergrund hat in kinderonkologischen Abteilungen die Prävention von Blutstrominfektionen, die von Gefäßkathetern ausgehen, einen sehr hohen Stellenwert (s. Fallbeispiel 3). Die Gesellschaft für Pädiatrische Onkologie und Hämatologie $(\mathrm{GPOH})$ hat seit 2001 Empfehlungen zum Umgang mit Port- und Broviac-Kathetern herausgegeben, die zuletzt 2013 aktualisiert wurden [84]. Dieser Beitrag soll zum einen über Studien in der pädiatrischen Onkologie berichten, in denen Präventionsbündel zur Reduktion der Inzidenz von Gefäßkatheter-assoziierten Blutstrominfektionen (CA-BSI) zum Einsatz kamen. Außerdem werden Vorschläge für ein solches Präventionsbündel gemacht, woran kinderonkologische Abteilungen ihr eigenes Vorgehen überprüfen können $[58,87]$.

\section{Präventionsbündel}

\section{Studien in der pädiatrischen Onkologie}

In > Tab. 1 sind die bis Ende 2015 publizierten Studien zusammengestellt, in denen Präventionsbündel in der Kinderonkologie zum Einsatz kamen. Bei pädiatrischen Intensivpflegepatienten mit nicht getunnelten zentralen Venenkathetern liegt ein Schwerpunkt von Präventionsbündeln auf der Anlage (Insertion) von zentralen

\section{TIPP FÜR DIE PRAXIS}

Präventionsbündel lassen sich vollständig und nachhaltig umsetzen, wenn

- sich die entsprechenden Maßnahmen gut in den Arbeitsablauf einpassen (Praktikabilität),

- genug angemessen qualifiziertes Pflegepersonal zur Verfügung steht,

- alle (auch Ärzte!) nach einem gemeinsam vereinbarten Standard geschult und trainiert werden (Wissen und Fähigkeiten; Einsatz von Trainingsdummies),

- eine Überprüfung der Compliance erfolgt.

Letzteres geschieht durch gegenseitige Kontrolle oder durch hiermit speziell beauftragtes Personal (z. B. Hygienefachpersonal). 
Venenkathetern (ZVK) $[66,69,70]$. Bei kinderonkologischen Patienten hingegen stehen Aspekte der Erhaltungspflege (maintenance care) im Vordergrund [58]. Implantiert wird der CVADi.d.R. im Operationsaal von einem Kinderchirurgen. Ein Teil aller kinderonkologischen Zentren (41\%) verabreicht bei diesem Eingriff eine perioperative Antibiotikaprophylaxe [87, 105].

\section{Ganzkörperwaschung}

In einigen Studien wird inzwischen auch eine Ganzkörperwaschung von kinderonkologischen Patienten mit Chlorhexidin-haltigen Waschtüchern empfohlen [26, 32]. Dieses aus der Intensivmedizin übernommene Vorgehen [28, 36, 46, 71] wird in Deutschland bisher vorwiegend zur Reduktion der Erregerdichte bzw. zur Dekolonisation bei Patienten eingesetzt, die mit Methicillin-resistenten S.aureus besiedelt sind $[14,56]$. Es ist bisher nicht klar, wie sich eine solche Strategie langfristig im Gesamtkonzept der Infektionsprävention bei hochgradig immunsupprimierten Kindern und Jugendlichen einordnen wird [55]. In den Studien von Choi et al. und Duffy et al. [26, 32] handelte es sich um Patientenkollektive mit einem hohen Anteil an allogen stammzelltransplantierten Kindern und Jugendlichen, bei denen möglicherweise die Chlorhexidin-Waschung (in Deutschland auch mit Octenidin-haltigen Waschlösungen/Tüchern möglich) eher zum Einsatz kommt als bei Patienten unter einer konventionellen Chemotherapie [26, 32].

\section{Prävention in der Praxis}

An dieser Stelle können nicht alle Aspekte der Prävention von CVAD-assoziierten Infektionen bei kinderonkologischen Patienten besprochen werden, hierzu wird auf die Empfehlungen der Fachgesellschaft $(\mathrm{GPOH})$ in der jeweils aktuellen Version verwiesen [84].

\section{Händedesinfektion (HD)}

Kinderonkologische Abteilungen sollten ihren Verbrauch an Händedesinfektionsmitteln mit den stationären Patiententagen korrelieren ( $3 \mathrm{ml}$ entsprechen dabei einer HD) $[57,74]$. Auf diese Weise erhält man zumindest einen orientierenden Hinweis auch im longitudinalen Verlauf über mehrere Jahre. Je mehr kinderonkologische Abteilungen an der „Aktion saubere Hände" teilnehmen, desto eher wird es möglich sein, nationale Referenzdaten für den HD-Verbrauch in dieser Hochrisikopopulation zu generieren (2015 lag der HDVerbrauch in der Kinderonkologie am Universitätsklinikum des Saarlandes bei 23 HD pro stationärem Patiententag).

\section{Hautantisepsis}

Zur Hautantisepsis vor Punktion eines Ports sollten Kombinationspräparate aus einem schnell wirksamen Alkohol (z.B. Isopropanol) und einem zweiten Wirkstoff mit Remanenzeffekt (Octenidin oder Chlorhexi-

\section{FALLBEISPIEL 3}

Ein 6-jähriger Junge mit akuter lymphoblastischer Leukämie hat zu Beginn der Chemotherapie einen Port-Katheter erhalten. Im Verlauf der Chemotherapie-Induktion wird er mit Fieber und starken Schmerzen über dem subkutan implantierten Port-Reservoir aufgenommen. Dort finden sich eine Rötung und eine fluktuierende Schwellung. Im Ultraschall zeigt sich eine Flüssigkeitsansammlung um das Port-Reservoir und eine erhöhte Echogenität der angrenzenden Weichteile. Die aktuelle Granulozytenzahl liegt bei $0,8 \times 10^{9} / \mathrm{I}$. In den periphervenös abgenommenen Blutkulturen wächst Staphylococcus aureus (Methicillin-sensibel). Der Port wird noch am Aufnahmetag chirurgisch explantiert. Intraoperativ entleert sich Eiter, in dem sich ebenfalls S. aureus nachweisen lässt. Ein primärer Wundverschluss ist aufgrund der ausgedehnten Entzündung nicht möglich.

Die empirische Therapie mit Piperacillin-Tazobactam und Teicoplanin wird nach Erhalt der mikrobiologischen Befunde und zwischenzeitlichem Anstieg der Granulozyten auf 1,5 x 109/1 auf Flucloxacillin i. v. umgestellt. Das Fieber sinkt am Tag 3 der i.v. Therapie. Die postoperativen Verbandswechsel müssen in Analgosedierung erfolgen. Die Port-Wunde zeigt erst nach Erholung der Granulozytenzahl eine langsame Heilungstendenz. Die Chemotherapie wird für eine Woche unterbrochen.

Der Patient wird 14 Tage stationär (i.v.) und anschließend 7 weitere Tage ambulant (p. o.) mit Flucloxacillin behandelt. Hinweise auf eine Endokarditis oder sonstige hämatogene Absiedlungen der Infektion finden sich nicht. Die Port-Tascheninfektion hinterlässt eine Narbe, die nach Ende der Chemotherapie eines weiteren kosmetisch-chirurgischen Eingriffs bedarf.

din) zum Einsatz kommen (Einwirkzeit $1 \mathrm{~min}$ ). Die antiseptische Behandlung der Broviac-Eintrittsstelle erfolgt i.d.R. mit Octenisept ${ }^{\circledR}$ (Octenidin plus Phenoxyethanol), weil dieses Präparat im Unterschied zu Antiseptika, die Isopropyl-Alkohol enthalten, kein Brennen an der Eintrittstelle verursacht $[47,95]$.

\section{Verbandswechsel}

Die Eintrittsstelle eines Broviac-Katheters ist eine wichtige Quelle für Infektionen, von denen eine sekundäre Bakteriämie oder eine Weichteilinfektion (des Kathetertunnels) ausgehen kann. Daher ist bei jedem Verbandswechsel eine antiseptische Behandlung der Eintrittsstelle erforderlich (nur sterile Tupfer und sterile Handschuhe oder eine Non-touch-Technik verwenden) [25].

\footnotetext{
Cave

Bestimmte Zytostatika (v. a. hoch dosiertes Methotrexat und Anthrazykline) können lokale Hautreaktionen am Broviac-Eintritt auslösen, die bei granulozytopenischen Patienten (kein Eiter!) klinisch schwer von Lokalinfektionen zu unterscheiden sind.
} 
- Tab. 1 Präventionsbündel-Studien in der Kinderonkologie.

\begin{tabular}{|c|c|c|c|c|}
\hline Studie & Methodik/Setting/Land & Station/Patienten / BSI & Wichtigste Strategien & Bündel erfolgreich? \\
\hline $\begin{array}{l}\text { Barrell et } \\
\text { al. } 2012 \\
{[13]}\end{array}$ & $\begin{array}{l}\text { Vor-/Nach-Intervention } \\
\text { (36 Monate) } \\
\text { CDC-Definitionen, } \\
\text { Inzidenzrate } \\
\text { Stammzelltransplantation } \\
\text { New York (Presbyterian } \\
\text { Hospital), USA }\end{array}$ & $\begin{array}{l}90 \text { Kinder und Jugendliche, } \\
\text { meist mit Trilumen-Broviac } \\
\text { (bis Tag 100) } \\
\mathbf{1 1 1} \text { BSI: } 41 \% \text { hospital- und } \\
59 \% \text { community-onset }\end{array}$ & $\begin{array}{l}\text { Standards für alle Aspekte } \\
\text { der Erhaltungspflege } \\
\text { Training an einem Dummy } \\
\text { Monitoring der Compliance }\end{array}$ & $\begin{array}{l}\text { Die IR sank von } 10 \text { auf } 3 \text { pro } \\
1000 \text { Anwendungstage } \\
\text { (gleich verteilt auf hospital- } \\
\text { und community-onset); vor } \\
\text { der Intervention deutlich } \\
\text { häufiger community-onset }\end{array}$ \\
\hline $\begin{array}{l}\text { Rinke et } \\
\text { al. } 2012 \\
{[76]}\end{array}$ & $\begin{array}{l}\text { unterbrochene Zeitserie } \\
\text { (prospektive Studie mit } 3 \\
\text { Beobachtungszeiträumen) } \\
10 \text { Monate vor sowie } 12 \text { und } \\
24 \text { Monate nach Interven- } \\
\text { tion } \\
\text { Johns Hopkins University, } \\
\text { Baltimore, USA }\end{array}$ & $\begin{array}{l}\text { Station mit } 18 \text { Betten } \\
\text { über } 200 \text { Neudiagnosen } \\
\text { und } 35 \text { Stammzelltrans- } \\
\text { plantationen pro Jahr }\end{array}$ & $\begin{array}{l}\text { sterile Handschuhe und } \\
\text { MNS bei Port-Punktion, } \\
\text { Systemanschluss/-wechsel, } \\
\text { Wechsel von NFCs } \\
\text { "scrub the hub" [45,61] } \\
\text { (Ethanol oder CHX) } \\
\text { Audits und öffentliche } \\
\text { Rückmeldung } \\
\text { monatliche Treffen }\end{array}$ & $\begin{array}{l}\text { Die IR der CA-BSIs sank von } \\
2,25 \text { auf } 0,81 \text { pro } 1000 \\
\text { CVAD-Tage in den zweiten } \\
12 \text { Monaten nach der Inter- } \\
\text { vention. } \\
\text { Dieser klinisch beeindru- } \\
\text { ckende Unterscheid war } \\
\text { statistisch nicht signifikant } \\
\text { (IRR: } 0,36 ; P=0,091 \text { ). In } \\
37 \% \text { aller CA-BSI musste der } \\
\text { CVAD explantiert werden. }\end{array}$ \\
\hline $\begin{array}{l}\text { Rinke et } \\
\text { al. } 2013 \\
{[75]}\end{array}$ & $\begin{array}{l}\text { unterbrochene Zeitserie } \\
\text { (prospektive Studie mit } \\
2 \text { Beobachtungszeit- } \\
\text { räumen) } \\
23 \text { Monate vor und } 24 \text { Mo- } \\
\text { nate nach Intervention } \\
\text { Johns Hopkins University, } \\
\text { Baltimore, USA }\end{array}$ & $\begin{array}{l}\text { ambulanter Versorgungs- } \\
\text { arm einer Station mit } 18 \\
\text { Betten } \\
\text { über } 200 \text { Neudiagnosen } \\
\text { und } 35 \text { Stammzelltrans- } \\
\text { plantationen pro Jahr }\end{array}$ & $\begin{array}{l}\text { Schulung aller Zielgruppen } \\
\text { nach dem Präventionsstan- } \\
\text { dard der Station (klinische } \\
\text { Mitarbeiter, Mitarbeiter in } \\
\text { der ambulanten Versor- } \\
\text { gung, Patienten und ihre } \\
\text { Familien) } \\
\text { Audits und Rückmeldung }\end{array}$ & $\begin{array}{l}\text { Die IR sank um } 48 \% \text { von } 0,63 \\
\text { auf } 0,32 \text { CA-BSI pro } 1000 \\
\text { CVAD-Tage ( } P=0,005) \text {. } \\
\text { Auch die mediane Rate der } \\
\text { Bakteriämien sank um } 54 \% \\
\text { von } 1,27 \text { auf } 0,59 \text { pro } 1000 \\
\text { CVAD-Tage }(P<0,001) \text {. }\end{array}$ \\
\hline $\begin{array}{l}\text { Berrueco } \\
\text { et al. } \\
2013 \text { [15] }\end{array}$ & $\begin{array}{l}\text { monozentrische Interventi- } \\
\text { onsstudie } \\
\text { ( } 36 \text { Monate) } \\
\text { CDC-Definitionen } \\
\text { Hospital Sant Joan de Deu, } \\
\text { University of Barcelona, } \\
\text { Spanien }\end{array}$ & $\begin{array}{l}8 \text { Betten Station plus } 8 \text { Bet- } \\
\text { ten Tagesklinik } \\
\text { alle neu aufgenommenen } \\
\text { Patienten mit Leukämie n= } \\
73 \\
\mathbf{3 6} \text { BSI, } 6 \text { BSI während der } \\
\text { Dauertherapie einer ALL } \\
\text { (Akute lymphoblastische } \\
\text { Leukämie) }\end{array}$ & $\begin{array}{l}\text { perioperative Antibiotika- } \\
\text { prophylaxe ( } 3 \text { Gaben) bei } \\
\text { Port-Implantation } \\
\text { Schulung / Training } \\
\text { Umstellung (erst im Verlauf } \\
\text { der Studie) von PVP-lod auf } \\
\text { CHX } 2 \%\end{array}$ & $\begin{array}{l}34 \% \text { der Patienten hatten } \\
\text { mind. } 1 \text { CA-BSI } \\
70 \% \text { stationär und } 30 \% \text { am- } \\
\text { bulant erworben. } \\
\text { Die IR wurde reduziert von } \\
6,7 \text { auf } 3,7 \text { und schließlich } \\
\text { auf } 1,5 \text { CA-BSI pro } 1000 \\
\text { Port-Anwendungstage } \\
(P=0,01) \text {. } \\
\text { CoNS in } 21 \text { von } 36=58 \% \\
\text { Explantation des Ports } 5 \text { von } \\
36=14 \%\end{array}$ \\
\hline $\begin{array}{l}\text { Choi et al. } \\
2013 \text { [26] }\end{array}$ & $\begin{array}{l}\text { Prä-/Post-Interventions- } \\
\text { studie } \\
(01 / 2005-04 / 2008 ; 05 / \\
2008-06 / 2011) ; \\
\text { CDC-Definition, mono- } \\
\text { zentrisch } \\
\text { University of Michigan, Ann } \\
\text { Arbor, Michigan, USA }\end{array}$ & $\begin{array}{l}26 \text { Betten } \\
\text { ca. } 100 \text { Neudiagnosen pro } \\
\text { Jahr, inkl. KMT-Service } \\
\text { Durch die Umstellung der } \\
\text { Definition für CA-BSI [44] } \\
\text { mussten } 18,6 \% \text { der vor } \\
2008 \text { diagnostizierten CA- } \\
\text { BSI ausgeschlossen werden. } \\
130 \text { BSI, davon } 70 \text { KMT }\end{array}$ & $\begin{array}{l}\text { schrittweise Implementie- } \\
\text { rung eines Präventionsbün- } \\
\text { dels inklusive: „scrub the } \\
\text { hub“ mit Ethanol, Ganzkör- } \\
\text { perwaschung / Duschen mit } \\
\text { CHX } \\
\text { patientennahe Checklisten } \\
{[21,107]}\end{array}$ & $\begin{array}{l}\text { Die ID wurde um } 45 \% \text { von } \\
2,9 \text { auf } 1,6 \text { pro } 1000 \\
\text { Patiententage reduziert } \\
\text { ( } P<0,004) \text {. Vor der Inter- } \\
\text { vention waren CoNS mit } \\
29 \% \text { die am häufigsten iso- } \\
\text { lierte Spezies). Im Verlauf } \\
\text { gab es keinen signifikanten } \\
\text { Unterschied mehr zwischen } \\
\text { KMT- und Nicht-KMT-Pa- } \\
\text { tienten }\end{array}$ \\
\hline $\begin{array}{l}\text { Duffy et } \\
\text { al. } 2015 \\
{[32]}\end{array}$ & $\begin{array}{l}\text { Prä-/Post-Interventions- } \\
\text { studie } \\
(01 / 2013-06 / 2013 ; 07 / \\
2013-01 / 2014) \\
\text { CDC-Definition, mono- } \\
\text { zentrisch } \\
\text { University of Michigan, Ann } \\
\text { Arbor, Michigan, USA }\end{array}$ & $\begin{array}{l}26 \text { Betten } \\
\text { ca. } 100 \text { Neudiagnosen pro } \\
\text { Jahr, inkl. KMT-Service } \\
\text { Das von Choi et al. etablier- } \\
\text { te Präventionsbündel [26] } \\
\text { wurde nach } 12 \text { Monaten } \\
\text { nicht mehr konsequent um- } \\
\text { gesetzt (Compliance). }\end{array}$ & $\begin{array}{l}\text { Forcierte Reimplementie- } \\
\text { rung des Präventionsbün- } \\
\text { dels von Choi et al. [26] } \\
\text { durch ein frisches multi- } \\
\text { disziplinäres Team. } \\
\text { Überprüfung der } \\
\text { Compliance }\end{array}$ & $\begin{array}{l}\text { Reduktion von } 8 \text { auf } 2 \text { CA- } \\
\text { BSI, statistisch nicht signifi- } \\
\text { kant aber nach Ansicht der } \\
\text { Autoren klinisch und öko- } \\
\text { nomisch relevant. Nur } 26 \% \\
\text { der Patienten erhielten die } \\
\text { eigentlich vorgesehene täg- } \\
\text { liche CHX-Waschung. }\end{array}$ \\
\hline
\end{tabular}


Tab. 1 (Fortsetzung)

\begin{tabular}{|c|c|c|c|c|}
\hline Studie & Methodik/Setting/Land & Station/Patienten / BSI & Wichtigste Strategien & Bündel erfolgreich? \\
\hline $\begin{array}{l}\text { Bundy et } \\
\text { al. } 2015 \\
{[23]}\end{array}$ & $\begin{array}{l}\text { Multizentrische Studie ab } \\
\text { November } 2009 \\
\text { CDC-Definitionen } \\
\text { Children's Hospital Asso- } \\
\text { ciation Hematology/Onco- } \\
\text { logy CLABSI Collaborative, } \\
\text { USA }\end{array}$ & $\begin{array}{l}32 \text { kinderonkologische } \\
\text { Zentren } \\
\text { Insgesamt wurden im Rah- } \\
\text { men dieser Studie ca. } 290 \\
\text { CA-BSI verhindert }\end{array}$ & $\begin{array}{l}\text { Sehr komplexes evidenzba- } \\
\text { siertes Präventionsbündel } \\
\text { für alle relevanten Proze- } \\
\text { duren. } \\
\text { Verantwortlichkeit für die } \\
\text { Umsetzung und für das } \\
\text { Monitoring der Compliance } \\
\text { (>80\%) bei den Teams vor } \\
\text { Ort }\end{array}$ & $\begin{array}{l}\text { internetbasiertes Erfas- } \\
\text { sungsmodul mit monat- } \\
\text { licher Aktualisierung der } \\
\text { Daten. } \\
\text { Reduktion der IR um } 28 \% \\
\text { von } 2,85 \text { auf } 2,04 \text { CA-BSI pro } \\
1000 \text { CVAD-Anwendungs- } \\
\text { tage (August 2012). } \\
\text { Relatives Risiko 0,71 (CI } 95 \% \\
0,55 \text { - 0,92) }\end{array}$ \\
\hline $\begin{array}{l}\text { Furt- } \\
\text { wängler } \\
\text { et al. } \\
2015 \text { [35] }\end{array}$ & $\begin{array}{l}\text { Vergleich von zwei } 21 \text { Mo- } \\
\text { nate langen prospektiven } \\
\text { Surveillance-Perioden (09/ } \\
2009-05 \text { /2013) } \\
\text { mod. CDC-Definitionen } \\
\text { [7,83], } \\
\text { monozentrisch, } \\
\text { Homburg / Saar, Deutsch- } \\
\text { land }\end{array}$ & $\begin{array}{l}12 \text { Betten Station, Tageskli- } \\
\text { nik, Ambulanz } \\
\text { ca. } 40 \text { - } 50 \text { Neudiagnosen } \\
\text { pro Jahr, } \\
\text { autologe Stammzelltrans- } \\
\text { plantation, } \\
84 \text { vs. } 81 \text { Patienten } \\
28 \text { BSI bei } 22 \text { Patienten } \\
\text { ( } 26 \% \text { aller Patienten mit } \\
\text { mind. } 1 \text { BSI) } \\
\text { vs. } 15 \text { BSI bei } 12 \text { Patienten } \\
\text { (15\%) }\end{array}$ & $\begin{array}{l}\text { Verbesserte Compliance bei } \\
\text { der Händehygiene } \\
\text { Octenisept }{ }^{\circledR} \text { am Broviac- } \\
\text { Eintritt, Octeniderm }{ }^{\circledR} \text { am } \\
\text { Hub und am Dreiwegehahn } \\
\text { selteneres Spülen des } \\
\text { Broviac CVAD (1-mal pro } \\
\text { Woche) } \\
\text { konfektionierte } \mathrm{NaCl} 0,9 \% \\
\text { Spülspritzen } \\
\text { Systemwechsel } 96 \mathrm{~h} \text { (+0,2 } \\
\mu \text { milter mit Endotoxin- } \\
\text { rückhalt) }\end{array}$ & $\begin{array}{l}\text { Reduktion der IR (alle BSI } \\
\text { pro } 1000 \text { stationäre Broviac- } \\
\text { Tage) von 7,76 auf 4,75. } \\
\text { Die Anzahl der BSI mit } \\
\text { Nachweis von CoNS wurde } \\
\text { von } 9 \text { BSI auf } 2 \text { reduziert } \\
\text { (IR 2,49 vs. } 0.63 \text { ). Die Un- } \\
\text { terschiede sind nicht statis- } \\
\text { tisch signifikant. } \\
\text { Die Investitionskosten } \\
\text { wurden bereits durch } \\
2 \text { verhinderte BSI pro Jahr } \\
\text { ausgeglichen. }\end{array}$ \\
\hline
\end{tabular}

\section{Folien- vs. Pflasterverband}

Bei klinischen Auffälligkeiten an der Eintrittsstelle sollte vor der antiseptischen Behandlung ein Wundabstrich erfolgen. Es gibt in der Kinderonkologie bisher keine randomisierten Studien, die transparenten Folienverbände mit konventionellen (sterilen!) Pflasterverbänden verglichen haben. Semipermeable Folienverbände haben praktische Vorteile: Die Eintrittsstelle lässt sich durch den Verband inspizieren und sie ist vor Feuchtigkeit von außen geschützt. Folienverbände können theoretisch bis zu 7 Tage genutzt werden (hier sind die Herstellerangaben wegweisend). Konventionelle Pflasterverbände sollte man alle $72 \mathrm{~h}$ wechseln und täglich palpieren (Schmerzen?).

\section{Nadel-Wechsel}

Die Huber-Nadel, mit der das subkutane Port-Reservoir punktiert wird, sollte nach 7-8 Tagen gewechselt werden. In jedem Fall ist eine zusätzliche Zugsicherung zu empfehlen.

\section{Unverträglichkeitsreaktion am Broviac-Katheter}

Ein grundsätzliches Problem bei kinderonkologischen Patienten ist die Dauer der Anwendung von Antiseptika und Pflasterverbänden am Broviac-Katheter. Diese Katheter liegen viele Monate, manchmal sogar länger als ein Jahr. Dies erhöht nach unserer Erfahrung das Risiko von Unverträglichkeitsreaktionen. Daher sollten z.B. Verbände mit integriertem Chlorhexidin-freisetzen- dem Pad oder Gelkissen [80, 96, 97] wenn möglich nur vorübergehend zum Einsatz kommen.

\section{Systemwechsel}

Die Vorbereitung des Infusionssystems, das meist aus mehreren Einzelkomponenten besteht, erfolgt unter aseptischen Kautelen nach der Händedesinfektion. Luer-Lock-Konnektionsstellen sind sehr sorgfältig festzudrehen. Einige Zentren nutzen zur Sicherheit patientennahe Rücklaufventile. Diese Ventile haben jedoch keinen infektionspräventiven Effekt [33]. Bei mobilen Patienten dürfen Infusionsleitungen nicht den Boden berühren. Der Systemwechsel soll im Regelfall nach 96 h erfolgen. Ausnahmen von dieser Regel sind Systeme, über die lipidhaltige parenterale Ernährungslösungen (nach $24 \mathrm{~h}$ ) oder Blutprodukte (nach 6h) verabreicht werden. Ivy et al. betonen zusätzlich die Notwendigkeit, Luer-Lock-Verbindungen vor einem Kontakt mit Wasser oder anderen nicht sterilen Flüssigkeiten/Sekreten zu schützen [48], weil Flüssigkeiten entlang des Gewindes (hydrostatischer Sog) in Richtung Katheterlumen vordringen können.

\section{Manipulation an Katheterhubs und Dreiwegehähnen}

Als „Hub“ wird das verstärkte, patientenferne Ende von Venenverweilkanülen, zentralen Gefäßkathetern und Verlängerungsleitungen an Huber-(Port-)Nadeln bezeichnet. Nadelfreie Konnektionsventile (NFC) ver- 
schließen einen Dreiweghahn, ein Verlängerungsstück oder den Katheterhub und erlauben das Ansetzen einer Spritze (z. B. zur Injektion oder zur Blutentnahme) ohne eine Injektionsnadel. Neben der Händedesinfektion wird vor jeder Manipulation an einem Gefäßkatheter die Desinfektion des Katheterhubs oder von Dreiwegehähnen empfohlen.

Merke

Die Kontamination von Hubs und Dreiwegehähnen ist eine wichtige Quelle von CR-BSI [65]. Wie genau eine solche Desinfektion erfolgen soll, wird kontrovers diskutiert.

Mit einem Alkoholtuch wird v. a. die äußere Oberfläche des Hubs/Dreiwegehahns erreicht [42]. Bei NFCs ist die Wischdesinfektion mit einem Alkoholtuch nach den Vorgaben des Herstellers die Standardmethode. Es ist auch möglich, den Hub/Dreiwegehahn mit einem geeigneten Antiseptikum einzusprühen. Dann sollten nach der Einwirkzeit (mind. 15s) unserer Meinung nach die Reste des Antiseptikums auf einer sterilen Kompresse „ausgeklopft“ werden. Einige Studien plädieren für den Einsatz von Chlorhexidin 2 \% (oder Octenidin $0,1 \%$ ) oder Isopropanol-haltigen Kombinationspräparaten [35]. Dies geschieht unter der Vorstellung, dass auch hier die Remanenzwirkung des Kombinationspräparats von Vorteil sein könnte [17,43,45, 79, 90]. Nach Blutentnahmen oder Bluttransfusionen sind Blutreste am Hub/Dreiwegehahn sehr sorgfältig zu entfernen (z.B. mit einer sterilen Kompresse, die mit einem Antiseptikum getränkt ist). Grundsätzlich ist es erforderlich, die Materialverträglichkeit der vor Ort eingesetzten Methode zur Desinfektion von Katheterhubs, Dreiwegehähnen und NFCs mit dem Hersteller der entsprechenden Medizinprodukte abzustimmen (Vermeiden von Haarrissen oder Materialbrüchen). Allerdings müssen diese Medizinprodukte mit einem alkoholischen Präparat desinfizierbar sein, ggf. ist sonst der Hersteller zu wechseln.

NFCs werden an Katheterhubs oder Dreiwegehähnen eingesetzt, an denen häufig manipuliert werden muss. Die Hersteller von NFCs müssen den Anwendern eine

\section{FAZIT FÜR DIE PRAXIS}

Der Umgang mit einem NFC kann das Risiko von CA-BSI erhöhen, wenn das Behandlungsteam nicht ausreichend in der korrekten Anwendung des speziellen Modells geschult ist oder nicht vor jedem Zugriff eine sorgfältige Desinfektion der NFC-Membran erfolgt [89]. Daher sollte in einer Abteilung in Absprache mit der Krankenhaushygiene immer das gleiche NFC-Modell zum Einsatz kommen [64, 100]. im Detail beschriebene und praktikable Methode der Desinfektion ihres NFC-Modells zur Verfügung stellen $[1,34]$.

\section{Inlinefilter}

Einige Abteilungen nutzen 0,2 $\mu \mathrm{m}$-Inlinefilter mit Endotoxinrückhalt. Deren infektionspräventiver Nutzen ist nicht bewiesen. Trotzdem sollte man ihren Einsatz erwägen, wenn Patienten komplexe Mischinfusionen zur parenteralen Ernährung erhalten und diese nicht unter Reinraumbedingungen in der Apotheke hergestellt werden [41]. Eine Partikelfiltration $(0,5 \mu \mathrm{m})$ von komplexen Mischinfusionen gilt als allgemein akzeptierter Standard $[49,50]$.

\section{Zubereiten von i. v. Arzneimitteln}

Kontaminierte Infusionslösungen bzw. i.v. Medikamente können eine BSI verursachen [10, 102]. Im manchmal hektischen Stationsalltag bedarf es auf Seiten des Pflegepersonals einer enormen Disziplin und Selbstkontrolle, um solche Kontaminationen durch ein strikt aseptisches Vorgehen bei der Rekonstitution und Zubereitung zu vermeiden. In der Praxis sind die in diesem Kontext auftretenden Hygieneprobleme allen Mitarbeitern bewusst, die i.v. zu verabreichende Arzneimittel rekonstituieren oder komplexe Mischinfusionen (z.B. zur parenteralen Ernährung) auf der Station unter ungünstigen Rahmenbedingungen zubereiten. Oft wird das Verwerfen von Resten aus Einzeldosisbehältnissen (Ampullen, Flaschen) kritisiert, weil dies „zu teuer sei“. Damit wird gegen die Vorgaben der Fachinformationen verstoßen („for single use only“) und die wichtige infektionspräventive Regel unterlaufen: „Pro Patient eine Ampulle/Spritze statt eine Ampulle/Spritze für mehrere Patienten!“.

Merke

In der Praxis ist die nicht sachgerechte (unzulässige) „Multidose“-Nutzung von Gebinden, die vom Hersteller zum 1-maligen Gebrauch deklariert sind, ein ernst zu nehmendes Problem (Teilentnahmen aus der gleichen Ampulle für mehrere Patienten) [9].

Die planbare Herstellung von komplexen Mischinfusionen zur parenteralen Ernährung sollte unter Reinraumbedingungen in der Klinikapotheke stattfinden [9]. Da für erwachsene Patienten und Jugendliche mit einem Körpergewicht über $40 \mathrm{~kg}$ fertig konfektionierte parenterale Ernährungslösungen kommerziell erhältlich sind, ist dies v. a. ein Problem in pädiatrischen/neonatologischen Kliniken.

\section{Blocken von CVADs}

In den meisten kinderonkologischen Zentren (90\%) werden CVADs, die nicht in Gebrauch sind, mit Heparin geblockt. Dazu soll ausschließlich patientenbezogenes Heparin aus Fertigampullen $(1 \mathrm{ml}=100 \mathrm{IE})$ zum Einsatz 
kommen. Reste aus diesen Ampullen sind sofort zu verwerfen. Bemerkenswert an dem bereits zitierten Review von Vonberg und Gastmeier [102] ist u. a., dass allein 30 von 128 analysierten Ausbrüchen durch kontaminierte Heparin-/NaCl 0,9\%-Spüllösungen verursacht wurden.

\section{Sterile Fertig-Spülspritzen ( $\mathrm{NaCl}$ 0,9\%)}

Um einen Verschluss des Katheters und chemische Unverträglichkeiten verschiedener i.v. Arzneimittel und Infusionslösungen zu vermeiden und (nach Blutentnahme oder Transfusion) Blutreste sorgfältig aus dem Katheterlumen zu entfernen, ist nach dem Verabreichen ein Spülen des Gefäßkatheters mit mindestens $10 \mathrm{ml}$ steriler Kochsalzlösung ohne Heparin-Zusatz [62] erforderlich. In der Kinderonkologie am Universitätsklinikum des Saarlandes geschieht dies (einschließlich der Ambulanz und Tagesklinik) ca. 15000-mal pro Jahr. Hierbei besteht ein nicht zu vernachlässigendes Kontaminationsrisiko [92], das sich möglicherweise durch den Einsatz vorkonfektionierter Spritzen verringern lässt, die sterile Kochsalzlösung ( $\mathrm{NaCl} 0,9 \%)$ enthalten $[16,72]$. Leider haben die kommerziell erhältlichen Spülspritzen den Nachteil unangenehmer Geschmacksensationen bei einigen Patienten, sodass die Kinder lernen, zwischen „gutem und bösem Kochsalz“ zu unterscheiden. Dieses Problem sollten die Hersteller unbedingt beseitigen. In entsprechend ausgestatteten Klinikapotheken können sterile Kochsalzspritzen zum Spülen von Gefäßkathetern auch automatisiert selbst hergestellt werden.

\section{Antimikrobielle Blocklösungen}

Eine gut untersuchte Möglichkeit, CR-BSI zu verhindern, ist das intermittierende Blocken des Katheters mit Taurolidin. Dies ist eine chemisch modifizierte, nicht toxische Aminosäure, die im Blutkreislauf zu Taurin verstoffwechselt wird. Taurolidin hat ein breites antimikrobielles Wirkspektrum [82, 99] bei einer minimalen Verweildauer im Katheterlumen von 4h [81]. Taurolidin kam bisher außerhalb der Onkologie v. a. in der Hämodialyse und bei heimparenteral ernährten Patienten zum Einsatz $[18,27,29,51,52,98,103]$. Auch in der Kinderonkologie gibt es Patienten, die vorübergehend oder anhaltend eine heimparenterale Ernährung erhalten müssen. Der Nutzen von Taurolidin wurde in 3 Metanalysen untersucht und aus infektionspräventiver Sicht positiv bewertet $[22,60,109]$. Leider verweigern viele Krankenkassen die Refinanzierung von Taurolidin-haltigen Blocklösungen im ambulanten Behandlungssektor, weil es auf der entsprechenden Liste der CE-zertifizierten Medizinprodukte des Gemeinsamen Bundesausschusses nicht gelistet ist. Damit wird den Patienten eine wichtige Präventionsmaßnahme von CR-BSI vorenthalten. Ethanol (80\%) wird vorwiegend zur adjuvanten Therapie von CR-BSI und seltener zur Prävention eingesetzt $[93,108]$.
Periphervenöse Venenverweilkanülen

Manchmal benötigen kinderonkologische Patienten mit einem CVADzusätzlich eine periphervenöse Venenverweilkanüle (PVK). Das Risiko von Infektionen, die von einem PVK ausgehen, wird von vielen Klinikern unterschätzt [40]. Eine von diesen Gefäßkathetern ausgehende Bakteriämie kann zu einer hämatogenen Besiedlung des CVAD (Katheterspitze) führen. Auch für die Anlage und Erhaltungspflege von PVKs sollte es einen eigenen infektionspräventiven Standard geben. Hierzu wird auf die entsprechende Empfehlung der KRINKO verwiesen.

Surveillance Zweifelsohne ist die prospektive Surveillance von CA-BSI ein wichtiges Instrument der internen Qualitätskontrolle [7,35,58,83]. Longitudinal nach dem gleichen Konzept erhobene Daten kann das Behandlungsteam zum Überprüfen des vor Ort etablierten Präventionsbündels nutzen [23]. Zielgrößen dabei sind z. B.

- Anzahl der Ereignisse (CA-BSI) pro 1000 stationäre Behandlungstage (Inzidenzdichte)

- Anzahl der Ereignisse (CA-BSI) pro 1000 stationäre Anwendungstage, wenn möglich getrennt nach Port oder Broviac (Inzidenzrate)

- Erreger- und Resistenzstatistik von Pathogenen, die in der Blutkultur isoliert wurden

- klinische Verlaufsdaten der CA-BSI-Ereignisse (z.B. Dauer des stationären Aufenthaltes, Notwendigkeit einer Intensivbehandlung, Explantation des CVAD usw.)

Ausgehend von den bis Ende 2015 vorgelegten Daten und Interpretationen der verfügbaren Studien hat eine Gruppe kinderonkologischer/kinderinfektiologischer Autoren gemeinsam mit Mitarbeitern des Nationalen Referenzzentrums für die Surveillance von nosokomialen Infektionen (Dr. Brar Piening, Frau Priv. Doz. Dr. Christine Geffers) einige Grundmerkmale für die Neukonzeption eines Erfassungsmoduls im KrankenhausInfektions-Surveillance-System (KISS) zur Surveillance von Blutstrominfektionen in der pädiatrischen Onkologie vorgeschlagen [86]. Allerdings ist die Diskussion über die finale Ausrichtung und inhaltliche Konzeption dieses Moduls noch nicht abgeschlossen. Es wäre auBerordentlich hilfreich, wenn in naher Zukunft allen in der Gesellschaft für Pädiatrische Onkologie und Hämatologie $(\mathrm{GPOH})$ organisierten Behandlungszentren ein einheitliches KISS-Erfassungsmodul für Blutstrominfektionen (mit oder ohne Bezug zum CVAD) bei kinderonkologischen Patienten zur Verfügung stünde. 


\section{KERNAUSSAGEN}

- Nur ein Teil der CA-BSI geht tatsächlich vom Gefäßkatheter aus und lässt sich durch gezielte Präventionsmaßnahmen verhindern [37,86].

- BSI und deren Therapie die Chemotherapie relevant verzögern, was sich möglicherweise ungünstig auf die Gesamtprognose auswirkt.

- Bei jedem Verbandswechsel ist eine antiseptische Behandlung der Eintrittsstelle des Broviac-Katheters nötig. Denn sie ist eine wichtige Quelle für Infektionen, von der eine sekundäre Bakteriämie oder eine Weichteilinfektion ausgehen kann.

- Bestimmte Zytostatika (v. a. hoch dosiertes Methotrexat und Anthrazykline) können lokale Hautreaktionen am BroviacEintritt auslösen, die bei granulozytopenischen Patienten (kein Eiter!) klinisch schwer von Lokalinfektionen zu unterscheiden sind.

- Die Kontamination von Hubs und Dreiwegehähnen ist eine wichtige Quelle von CR-BSI [65]. Der Umgang mit einem NFC erfordert daher ein gut geschultes Behandlungsteam und die vor jedem Zugriff sorgfältige Desinfektion der NFC-Membran [89].

- Kontaminierte Infusionslösungen bzw. i.v. Medikamente können eine BSI verursachen $[10,102]$. In der Praxis ist die nicht sachgerechte (unzulässige) Teilentnahme aus der gleichen Ampulle für mehrere Patienten ein ernstes Problem [9].

- Zum Blocken von CVAD soll ausschließlich patientenbezogenes Heparin aus Fertigampullen zum Einsatz kommen.

- Durch intermittierendes Blocken des Katheters mit Taurolidin lassen sich CR-BSI vermeiden.

- Eine von PVKs ausgehende Bakteriämie kann zu einer hämatogenen Besiedlung des CVAD führen. Auch für die Anlage und Erhaltungspflege von PVKs sollte es einen eigenen infektionspräventiven Standard geben.

- Ein einheitliches KISS-Erfassungsmodul für Blutstrominfektionen (mit oder ohne Bezug zum CVAD) bei kinderonkologischen Patienten ist wünschenswert.

\section{Interessenkonflikte}

Die Autoren geben an, dass kein Interessenskonflikt besteht.

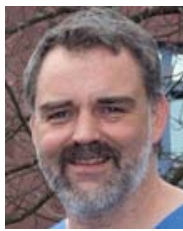

\section{Arne Simon}

Prof. Dr. med., Pädiatrischer Onkologe, Hämatologe und Infektiologe am Universitätsklinikum des Saarlandes in Homburg. Seit 2004 ist er berufenes Mitglied der Kommission für Krankenhaushygiene und Infektionsprävention beim Robert

Koch-Institut, Berlin. Er ist Koordinator der KRINKO-Arbeitsgruppe Prävention von Infektionen, die von Gefäßkathetern ausgehen.

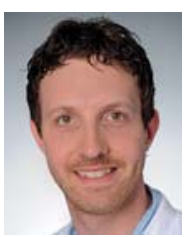

\section{Max Scheler}

Pädiatrischer Onkologe am Klinikum der Universität zu Köln. Neben seiner klinischen Tätigkeit in der Abteilung für Kinderonkologie und -hämatologie ist er Mitglied der Arbeitsgemeinschaft „Infektionen" der Gesellschaft für Pädiatrische Onkologie und Hämatologie und beteiligt sich an der Erarbeitung von Leitlinien und evidenzbasierten Empfehlungen zu infektiologischen Themen in der Behandlung pädiatrisch-onkologischer Patienten.

\section{Korrespondenzadresse}

Prof. Dr. med. Arne Simon

Klinik für Pädiatrische Onkologie und Hämatologie Universitätsklinikum des Saarlandes

Kirrberger Straße, Gebäude 9

66421 Homburg/Saar

E-Mail: Arne.Simon@uks.eu

\section{Literaturverzeichnis}

[1] Adams D, Karpanen T, Worthington T et al. Infection risk associated with a closed luer access device. J Hosp Infect 2006; 62: $353-357$

[2] Adler A, Yaniv I, Solter E et al. Catheter-associated bloodstream infections in pediatric hematology-oncology patients: factors associated with catheter removal and recurrence. J Pediatr Hematol Oncol 2006; 28: 23 -28

[3] Adler A, Yaniv I, Steinberg R et al. Infectious complications of implantable ports and Hickman catheters in paediatric haematology-oncology patients. J Hosp Infect 2006; 62: $358-365$

[4] Allen RC, Holdsworth MT, Johnson CA et al. Risk determinants for catheter-associated blood stream infections in children and young adults with cancer. Pediatr Blood Cancer 2008; 51: $53-58$

[5] Ammann RA, Bodmer N, Hirt A et al. Predicting adverse events in children with fever and chemotherapy-induced neutropenia: the prospective multicenter SPOG 2003 FN study. J Clin Oncol 2010; 28: 2008-2014 
[6] Ammann RA, Hirt A, Luthy AR et al. Predicting bacteremia in children with fever and chemotherapy-induced neutropenia. Pediatr Infect Dis J 2004; 23: 61 - 67

[7] Ammann RA, Laws H], Schrey D et al. Bloodstream infection in paediatric cancer centres-leukaemia and relapsed malignancies are independent risk factors. Eur J Pediatr 2015; 174: $675-686$

[8] Ammann RA, Tissing WJ, Phillips B. Rationalizing the approach to children with fever in neutropenia. Curr Opin Infect Dis 2012; 25: 258-265

[9] Arbeitsgruppe KRINKO-BfArM-RKI. Bericht der Arbeitsgruppe KRINKO-BfArM-RKI: Zu spezifischen Fragen bezüglich Rekonstitution, Zubereitung und Applikation von Arzneimitteln und Infusionslösungen sowie zur Hautantiseptik. Berlin: Epidemiol Bulletin des Robert Koch-Instituts; 2016: Nr. $20173-178$

[10] Austin PD, Elia M. A systematic review and meta-analysis of the risk of microbial contamination of aseptically prepared doses in different environments. J Pharm Pharm Sci 2009; 12: $233-242$

[11] Averbuch D, Cordonnier C, Livermore DM et al. Targeted therapy against multi-resistant bacteria in leukemic and hematopoietic stem cell transplant recipients: guidelines of the 4th European Conference on Infections in Leukemia (ECIL-4, 2011). Haematologica 2013; 98: 1836 - 1847

[12] Averbuch D, Orasch C, Cordonnier C et al. European guidelines for empirical antibacterial therapy for febrile neutropenic patients in the era of growing resistance: summary of the 2011 4th European Conference on Infections in Leukemia. Haematologica 2013; 98: 1826-1835

[13] Barrell C, Covington L, Bhatia M et al. Preventive strategies for central line-associated bloodstream infections in pediatric hematopoietic stem cell transplant recipients. Am J Infect Control 2012; 40: 434-439

[14] Bass P, Karki S, Rhodes D et al. Impact of chlorhexidine-impregnated washcloths on reducing incidence of vancomycin-resistant enterococci colonization in hematology-oncology patients. Am J Infect Control 2013; 41: 345 - 348

[15] Berrueco R, Rives S, Catala A et al. Prospective Surveillance Study of Blood Stream Infections Associated With Central Venous Access Devices (Port-type) in Children With Acute Leukemia: An Intervention Program. J Pediatr Hematol Oncol 2013; 35: e194-e199

[16] Bertoglio S, Rezzo R, Merlo FD et al. Pre-filled normal saline syringes to reduce totally implantable venous access deviceassociated bloodstream infection: a single institution pilot study. J Hosp Infect 2013; 84: 85-88

[17] Bishay M, Retrosi G, Horn V et al. Chlorhexidine antisepsis significantly reduces the incidence of sepsis and septicemia during parenteral nutrition in surgical infants. J Pediatr Surg 2011; 46: $1064-1069$

[18] Bisseling TM, Willems MC, Versleijen MW et al. Taurolidine lock is highly effective in preventing catheter-related bloodstream infections in patients on home parenteral nutrition: a heparin-controlled prospective trial. Clin Nutr 2010; 29: $464-468$

[19] Biwersi C, Hepping N, Bode U et al. Bloodstream infections in a German paediatric oncology unit: Prolongation of inpatient treatment and additional costs. Int J Hyg Environ Health 2009; 212: 541 - 546

[20] Blijlevens NM, Donnelly JP, De Pauw BE. Mucosal barrier injury: biology, pathology, clinical counterparts and consequences of intensive treatment for haematological mali- gnancy: an overview. Bone Marrow Transplant 2000; 25: $1269-1278$

[21] Bosk CL, Dixon-Woods M, Goeschel CA et al. Reality check for checklists. Lancet 2009; 374: 444-445

[22] Bradshaw JH, Puntis JW. Taurolidine and catheter-related bloodstream infection: a systematic review of the literature. J Pediatr Gastroenterol Nutr 2008; 47: 179-186

[23] Bundy DG, Gaur AH, Billett AL et al. Preventing CLABSIs among pediatric hematology/oncology inpatients: national collaborative results. Pediatrics 2014; 134: e1678-e1685

[24] Cheng S, Teuffel O, Ethier MC et al. Health-related quality of life anticipated with different management strategies for paediatric febrile neutropaenia. Br J Cancer 2011; 105: $606-611$

[25] Chesshyre E, Goff Z, Bowen A et al. The prevention, diagnosis and management of central venous line infections in children. J Infect 2015; 71: (Suppl. 01): 59-75

[26] Choi SW, Chang L, Hanauer DA et al. Rapid reduction of central line infections in hospitalized pediatric oncology patients through simple quality improvement methods. Pediatr Blood Cancer 2013; 60: 262-269

[27] Chu HP, Brind J, Tomar R et al. Significant Reduction in Central Venous Catheter-related Bloodstream Infections in Children on HPN After Starting Treatment With Taurolidine Line Lock. J Pediatr Gastroenterol Nutr 2012; 55: 403-407

[28] Climo MW, Yokoe DS, Warren DK et al. Effect of daily chlorhexidine bathing on hospital-acquired infection. $N$ Engl ] Med 2013; 368: $533-542$

[29] Cullis PS, McKee RF. Taurolidine lock - experience from the West of Scotland. Clin Nutr 2011; 30: 399-400

[30] Deutsche Gesellschaft für pädiatrische Infektiologie (DGPI), Gesellschaft für Pädiatrische Onkologie und Hämatologie $(\mathrm{GPOH})$. Diagnostik und Therapie bei Kindern mit onkologischer Grunderkrankung, Fieber und Granulozytopenie (mit febriler Neutropenie) außerhalb der allogenen Stammzelltransplantation. Arbeitsgemeinschaft Wissenschaftlicher Fachgesellschaften (AWMF); 2016: Registernummer 048/14

[31] Diorio C, Martino J, Boydell KM et al. Parental perspectives on inpatient versus outpatient management of pediatric febrile neutropenia. J Pediatr Oncol Nurs 2011; 28: 355 362

[32] Duffy EA, Rodgers CC, Shever LL et al. Implementing a Daily Maintenance Care Bundle to Prevent Central Line-Associated Bloodstream Infections in Pediatric Oncology Patients. J Pediatr Oncol Nurs 2015; 32: $394-400$

[33] Ellger B, Kiski D, Diem E et al. Non-return valves do not prevent backflow and bacterial contamination of intravenous infusions. J Hosp Infect 2011; 78: 31 - 35

[34] Engelhart S, Exner M, Simon A. In vitro study on the disinfectability of two split-septum needle-free connection devices using different disinfection procedures. GMS hygiene and infection control 2015; 10: Doc17 DOI 10.3205/ dgkh000260

[35] Furtwangler R, Laux C, Graf N et al. Impact of a modified Broviac maintenance care bundle on bloodstream infections in paediatric cancer patients. GMS hygiene and infection control 2015; 10: Doc15 DOI 10.3205/dgkh000258

[36] Gastmeier P, Kampf KP, Behnke M et al. An observational study of the universal use of octenidine to decrease nosocomial bloodstream infections and MDR organisms. J Antimicrob Chemother 2016; 71: 2569-2576 
[37] Gaur AH, Bundy DG, Gao C et al. Surveillance of hospitalacquired central line-associated bloodstream infections in pediatric hematology-oncology patients: lessons learned, challenges ahead. Infect Control Hosp Epidemiol 2013; 34: $316-320$

[38] Gyssens IC, Kern WV, Livermore DM. The role of antibiotic stewardship in limiting antibacterial resistance among hematology patients. Haematologica 2013; 98: 1821 - 1825

[39] Handrup MM, Moller JK, Frydenberg M et al. Placing of tunneled central venous catheters prior to induction chemotherapy in children with acute lymphoblastic leukemia. Pediatr Blood Cancer 2010; 55: 309-313

[40] Helm RE, Klausner JD, Klemperer JD et al. Accepted but unacceptable: peripheral IV catheter failure. J Infus Nurs 2015; 38: 189-203

[41] Herbig S, Kaiser V, Maurer J et al. ADKA-Leitlinie: Aseptische Herstellung und Prüfung applikationsfertiger Parenteralia. Krankenhauspharmazie 2013; 34: 93-106

[42] Holroyd JL, Paulus DA, Rand KH et al. Universal intravenous access cleaning device fails to sterilize stopcocks. Anesth Analg 2014; 118: $333-343$

[43] Hong H, Morrow DF, Sandora T] et al. Disinfection of needleless connectors with chlorhexidine-alcohol provides longlasting residual disinfectant activity. Am J Infect Control 2013; 41: e77 - e79

[44] Horan TC, Andrus M, Dudeck MA. CDC/NHSN surveillance definition of health care-associated infection and criteria for specific types of infections in the acute care setting. Am J Infect Control 2008; 36: 309-332

[45] Horvath B, Norville R, Lee D et al. Reducing central venous catheter-related bloodstream infections in children with cancer. Oncol Nurs Forum 2009; 36: 232-238

[46] Huang SS, Septimus E, Kleinman K et al. Targeted versus universal decolonization to prevent ICU infection. N Engl J Med 2013; 368: 2255-2265

[47] Hubner NO, Siebert J, Kramer A. Octenidine dihydrochloride, a modern antiseptic for skin, mucous membranes and wounds. Skin Pharmacol Physiol 2010; 23: 244-258

[48] Ivy DD, Calderbank M, Wagner BD et al. Closed-hub systems with protected connections and the reduction of risk of catheter-related bloodstream infection in pediatric patients receiving intravenous prostanoid therapy for pulmonary hypertension. Infect Control Hosp Epidemiol 2009; 30: $823-829$

[49] Jack T, Boehne M, Brent BE et al. In-line filtration reduces severe complications and length of stay on pediatric intensive care unit: a prospective, randomized, controlled trial. Intensive Care Med 2012; 38: 1008 - 1016

[50] Jack T, Brent BE, Boehne M et al. Analysis of particulate contaminations of infusion solutions in a pediatric intensive care unit. Intensive Care Med 2010; 36: 707 - 711

[51] Jurewitsch B, Jeejeebhoy KN. Taurolidine lock: the key to prevention of recurrent catheter-related bloodstream infections. Clin Nutr 2005; 24: $462-465$

[52] Jurewitsch B, Lee T, Park J et al. Taurolidine $2 \%$ as an antimicrobial lock solution for prevention of recurrent catheterrelated bloodstream infections. JPEN J Parenter Enteral Nutr 1998; 22: 242-244

[53] Kelly M, Conway M, Wirth K et al. Moving CLABSI prevention beyond the intensive care unit: risk factors in pediatric oncology patients. Infect Control Hosp Epidemiol 2011; 32: $1079-1085$
[54] Kelly M], Vivier PM, Panken TM et al. Bacteremia in febrile nonneutropenic pediatric oncology patients. Pediatr Blood Cancer 2010; 54: 83-87

[55] Kommission für Krankenhaushygiene und Infektionsprävention beim Robert Koch-Institut (KRINKO). Empfehlungen zu den Anforderungen an die Hygiene bei der medizinischen Versorgung von immunsupprimierten Patienten. Bundesgesundheitsbl - Gesundheitsforsch - Gesundheitsschutz 2010; 53: 357-388

[56] Kommission für Krankenhaushygiene und Infektionsprävention beim Robert Koch-Institut (KRINKO). Empfehlungen zur Prävention und Kontrolle von Methicillin-resistenten Staphylococcus aureus-Stämmen (MRSA) in medizinischen und pflegerischen Einrichtungen. Bundesgesundheitsbl Gesundheitsforsch - Gesundheitsschutz 2014; 57: 696 732

[57] Kommission für Krankenhaushygiene und Infektionsprävention beim Robert Koch-Institut (KRINKO). Empfehlungen zur Händehygiene in Einrichtungen des Gesundheitswesens. Bundesgesundheitsbl - Gesundheitsforsch - Gesundheitsschutz 2016; 59: 1189-1220

[58] Krenn T, Fleischhack G, Moser O et al. Bloodstream infections in paediatric cancer patients. Prospective comparative study in 2 university hospitals. Klin Padiatr 2011; 223: 335 340

[59] Lehrnbecher T, Phillips R, Alexander S et al. Guideline for the Management of Fever and Neutropenia in Children With Cancer and/or Undergoing Hematopoietic Stem-Cell Transplantation. J Clin Oncol 2012; 30: 4427-4438

[60] Liu H, Liu H, Deng J et al. Preventing catheter-related bacteremia with taurolidine-citrate catheter locks: a systematic review and meta-analysis. Blood purification 2014; 37: $179-187$

[61] Lockman JL, Heitmiller ES, Ascenzi JA et al. Scrub the hub! Catheter needleless port decontamination Anesthesiology 2011; 114: 958

[62] Lopez-Briz E, Ruiz-Garcia V. [Effectiveness of heparin versus $\mathrm{NaCl} 0.9 \%$ in central venous catheter flushing. A systematic review]. Farm Hosp 200529: 258-264

[63] Lukac PJ, Bonomo RA, Logan LK. Extended-spectrum betalactamase-producing Enterobacteriaceae in children: Old Foe, Emerging Threat. Clin Infect Dis 2015; 60: DOI $10.1093 /$ cid/civ020

[64] Maragakis LL, Bradley KL, Song X et al. Increased catheterrelated bloodstream infection rates after the introduction of a new mechanical valve intravenous access port. Infect Control Hosp Epidemiol 2006; 27: 67 - 70

[65] Marschall J, Mermel LA, Fakih M et al. Strategies to prevent central line-associated bloodstream infections in acute care hospitals: 2014 update. Infect Control Hosp Epidemiol 2014; 35: $753-771$

[66] McMullan C, Propper G, Schuhmacher C et al. A multidisciplinary approach to reduce central line-associated bloodstream infections. Jt Comm J Qual Patient Saf 2013; 39: 61 69

[67] Meckler G, Lindemulder S. Fever and neutropenia in pediatric patients with cancer. Emerg Med Clin North Am 2009; 27: $525-544$

[68] Miedema KG, Winter RH, Ammann RA et al. Bacteria causing bacteremia in pediatric cancer patients presenting with febrile neutropenia-species distribution and susceptibility patterns. Support Care Cancer 2013; 21: 2417-2426 
[69] Miller MR, Griswold M, Harris JM et al. Decreasing PICU catheter-associated bloodstream infections: NACHRI's quality transformation efforts. Pediatrics 2010; 125: 206 - 213

[70] Miller MR, Niedner MF, Huskins WC et al. Reducing PICU central line-associated bloodstream infections: 3-year results. Pediatrics 2011; 128: e1077-1083

[71] Milstone AM, Elward A, Song X et al. Daily chlorhexidine bathing to reduce bacteraemia in critically ill children: a multicentre, cluster-randomised, crossover trial. Lancet 2013; 381: 1099-1106

[72] Pohl F, Hartmann W, Holzmann T et al. Risk of infection due to medical interventions via central venous catheters or implantable venous access port systems at the middle port of a three-way cock: luer lock cap vs. luer access split septum system (Q-Syte). BMC Infect Dis 2014; 14: 41

[73] Raymond J, Aujard Y. Nosocomial infections in pediatric patients: a European, multicenter prospective study. European Study Group. Infect Control Hosp Epidemiol 2000; 21: $260-263$

[74] Reichardt C, Koniger D, Bunte-Schonberger K et al. Three years of national hand hygiene campaign in Germany: what are the key conclusions for clinical practice? J Hosp Infect 2013; 83: (Suppl. 01): S11-S16

[75] Rinke ML, Bundy DG, Chen AR et al. Central line maintenance bundles and CLABSIs in ambulatory oncology patients. Pediatrics 2013; 132: e1403-e1412

[76] Rinke ML, Chen AR, Bundy DG et al. Implementation of a central line maintenance care bundle in hospitalized pediatric oncology patients. Pediatrics 2012; 130: e996-e1004

[77] Samet A, Sledzinska A, Krawczyk B et al. Leukemia and risk of recurrent Escherichia coli bacteremia: genotyping implicates $\mathrm{E}$. coli translocation from the colon to the bloodstream. Eur J Clin Microbiol Infect Dis 2013; 32: $1393-1400$

[78] Sammons JS, Localio R, Xiao R et al. Clostridium difficile infection is associated with increased risk of death and prolonged hospitalization in children. Clin Infect Dis 2013; 57: $1-8$

[79] Sannoh S, Clones B, Munoz J et al. A multimodal approach to central venous catheter hub care can decrease catheterrelated bloodstream infection. Am J Infect Control 2010; 38: $424-429$

[80] Scheithauer S, Lewalter K, Schroder ] et al. Reduction of central venous line-associated bloodstream infection rates by using a chlorhexidine-containing dressing. Infection 2014; 42: 155-159

[81] Schlicht A, Fleischhack G, Herdeis C et al. In vitro investigation of the exposure time necessary to yield a 5 log reduction of clinicall relevant bacteria by a taurolidine containing antimicrobial catheter lock solution. Hygiene \& Medizin 2009; 34: $343-345$

[82] Shah CB, Mittelman MW, Costerton JW et al. Antimicrobial activity of a novel catheter lock solution. Antimicrob Agents Chemother 2002; 46: 1674-1679

[83] Simon A, Ammann RA, Bode U et al. Nosocomial infections in pediatric cancer patients: results of a prospective surveillance study from 7 University hospitals in Germany and Switzerland. BMC Infect Dis 2008; 70: DOI 10.1186/14712334-8-70

[84] Simon A, Beutel K, Trautmann M et al. Evidenzbasierte Empfehlungen zur Anwendung dauerhaft implantierter, zentralvenöser Zugänge in der pädiatrischen Onkologie. 4. Aufl. Wiesbaden: mhp; 2013
[85] Simon A, Fleischhack G, Hasan C et al. Surveillance for nosocomial and central line-related infections among pediatric hematology-oncology patients. Infect Control Hosp Epidemiol 2000; 21: 592 - 596

[86] Simon A, Furtwangler R, Graf N et al. Surveillance of bloodstream infections in pediatric cancer centers - what have we learned and how do we move on? GMS hygiene and infection control 2016; 11: Doc11 DOI 10.3205/ dgkh000271

[87] Simon A, Graf N, Furtwangler R. Results of a Multicentre Survey Evaluating Clinical Practice of Port and Broviac Management in Paediatric Oncology. Klin Padiatr 2013; 225: $145-151$

[88] Simon A, Groger N, Wilkesmann A et al. Restricted use of glycopeptides in paediatric cancer patients with fever and neutropenia. Int J Antimicrob Agents 2006; 28: 417 - 422

[89] Smith JS, Kirksey KM, Becker H et al. Autonomy and Self-efficacy as Influencing Factors in Nurses' Behavioral Intention to Disinfect Needleless Intravenous Systems. J Infus Nurs 2011; 34: $193-200$

[90] Soothill JS, Bravery K, Ho A et al. A fall in bloodstream infections followed a change to $2 \%$ chlorhexidine in $70 \%$ isopropanol for catheter connection antisepsis: a pediatric single center before/after study on a hemopoietic stem cell transplant ward. Am J Infect Control 2009; 37: 626-630

[91] Steinberg JP, Robichaux C, Tejedor SC et al. Distribution of pathogens in central line-associated bloodstream infections among patients with and without neutropenia following chemotherapy: evidence for a proposed modification to the current surveillance definition. Infect Control Hosp Epidemiol 2013; 34: 171 - 175

[92] Stucki C, Sautter AM, Favet J et al. Microbial contamination of syringes during preparation: the direct influence of environmental cleanliness and risk manipulations on end-product quality. Am J Health Syst Pharm 2009; 66: 2032-2036

[93] Tan M, Lau J, Guglielmo BJ. Ethanol locks in the prevention and treatment of catheter-related bloodstream infections. Ann Pharmacother 2014; 48: 607-615

[94] Teuffel O, Amir E, Alibhai SM et al. Cost-effectiveness of outpatient management for febrile neutropenia in children with cancer. Pediatrics 2011; 127: e279-286

[95] Tietz A, Frei R, Dangel M et al. Octenidine hydrochloride for the care of central venous catheter insertion sites in severely immunocompromised patients. Infect Control Hosp Epidemiol 2005; 26: $703-707$

[96] Timsit JF, Mimoz O, Mourvillier B et al. Randomized controlled trial of chlorhexidine dressing and highly adhesive dressing for preventing catheter-related infections in critically ill adults. Am J Respir Crit Care Med 2012; 186: 1272 1278

[97] Timsit JF, Schwebel C, Bouadma L et al. Chlorhexidine-impregnated sponges and less frequent dressing changes for prevention of catheter-related infections in critically ill adults: a randomized controlled trial. Jama 2009; 301: $1231-1241$

[98] Toure A, Lauverjat M, Peraldi C et al. Taurolidine lock solution in the secondary prevention of central venous catheterassociated bloodstream infection in home parenteral nutrition patients. Clin Nutr 2012; 31: 567-570

[99] Traub WH, Leonhard B, Bauer D. Taurolidine: in vitro activity against multiple-antibiotic-resistant, nosocomially significant clinical isolates of Staphylococcus aureus, Enterococcus faecium, and diverse Enterobacteriaceae. Chemotherapy $1993 ; 39: 322-330$ 
[100] Trautmann M, Kreutzberger M, Bobic R et al. Disinfection of a needleless connector with alcohol-based disinfectant wipes - an experimental study. Hygiene \& Medizin 2012; 37: $354-359$

[101] van der Velden W], Herbers AH, Netea MG et al. Mucosal barrier injury, fever and infection in neutropenic patients with cancer: introducing the paradigm febrile mucositis. Br J Haematol 2014; 167: $441-452$

[102] Vonberg RP, Gastmeier P. Hospital-acquired infections related to contaminated substances. J Hosp Infect 2007; 65: $15-23$

[103] Wanten GJA, Bisseling TM. Responding letter to editor Taurolidine lock is highly effective in preventing catheterrelated bloodstream infections in patients on home parenteral nutrition: a heparin-controlled prospective trial. Clin Nutr 2011; 30: 401-401

[104] Weichert S, Simon A, von Müller L et al. Clostridium-difficile-assoziierte Infektionen im Kindes- und Jugendalter. Monatsschrift für Kinderheilkunde 2015; 163: 427-436

[105] Weiss K, Simon A, Graf N et al. Clinical Practice Audit: Perioperative Antibiotic Prophylaxis in Paediatric Cancer Patients with Broviac Catheter Implantation. Klin Padiatr 2016; 228: 139-144
[106] Wilson MZ, Rafferty C, Deeter D et al. Attributable costs of central line-associated bloodstream infections in a pediatric hematology/oncology population. Am J Infect Control 2014; 42: $1157-1160$

[107] Winters BD, Gurses AP, Lehmann $\mathrm{H}$ et al. Clinical review: checklists - translating evidence into practice. Crit Care 2009; 13: 210

[108] Wolf J, Shenep JL, Clifford V et al. Ethanol lock therapy in pediatric hematology and oncology. Pediatr Blood Cancer 2013; 60: $18-25$

[109] Zacharioudakis IM, Zervou FN, Arvanitis M et al. Antimicrobial Lock Solutions as a Method to Prevent Central LineAssociated Bloodstream Infections: A Meta-analysis of Randomized Controlled Trials. Clin Infect Dis 2014; 59: $1741-1749$

Bibliografie

DOI http://dx.doi.org/10.1055/s-0042-123726 Krankenhaushygiene up2date 2017; 12: 43-56 (c) Georg Thieme Verlag KG Stuttgart · New York ISSN 1862-5797 


\section{Punkte sammeln auf CME. thiemede}

Diese Fortbildungseinheit ist 12 Monate online für die Teilnahme verfügbar.

Sollten Sie Fragen zur Online-Teilnahme haben, finden Sie unter http://cme.thieme.de|hilfe eine ausführliche Anleitung. Wir wünschen viel Erfolg beim Beantworten

der Fragen!

Unter eref.thieme.de/ZZZWHQ74 oder über den QR-Code kommen Sie direkt zum Artikel zur Eingabe der Antworten.

VNR 2760512017152374684

\section{Frage 1}

Welche Gefäßkatheter kommen bei kinderonkologischen Patienten vorrangig zum Einsatz?

A periphervenöse Verweilkanülen

B periphervenös angelegte zentrale Venenkatheter

C konventionelle (nicht getunnelte) zentrale Venenkatheter

D konventionelle zentrale Venenkatheter mit antimikrobieller Beschichtung

E dauerhaft implantierte zentrale Venenkatheter vom Typ Broviac oder Port

\section{Frage 2}

Welche Aussage zu Blutstrominfektionen (mit positiver Blutkultur) treffen für kinderonkologische Patienten unter einer intensiven Chemotherapie zu?

A Blutstrominfektionen (BSI), die durch bakterielle Infektionserreger ausgelöst werden, machen in der Kinderonkologie ca. $50 \%$ aller nosokomialen Infektionen mit Erregernachweis aus.

B Bei Fieber und Granulozytopenie findet man in $50 \%$ der Fälle eine positive Blutkultur.

C Blutstrominfektionen erfordern immer eine mindestens 14-tägige stationäre Behandlung.

D Blutkulturen werden immer nur aus einer peripheren Vene abgenommen, nicht aus dem Port- oder Broviac-Katheter.

E Die Quelle der in der Blutkultur nachgewiesenen Erreger ist meistens der Port- oder Broviac-Katheter.

\section{Frage 3}

Welcher grampositive Erreger lässt sich besonders häufig bei kinderonkologischen Patienten mit einer ausgeprägten Mukositis in der Blutkultur nachweisen (z. B. bei akuter myeloischer Leukämie nach hoch dosiertem Cytarabin)?

A Staphylococcus aureus,

B Enterococcus faecium

C vergrünende Streptokokken

D Koagulase-negative Staphylokokken

E Lactobacillus rhamnosus

\section{Frage 4}

Welche Aussage zu BSI (mit positiver Blutkultur) trifft für kinderonkologische Patienten unter einer intensiven Chemotherapie nicht zu?

A BSI können in Phasen der ausgeprägten Immunsuppression das Leben der Patienten akut gefährden.

B Die mit der Behandlung von BSI verbunden Mehrkosten sind vor dem Hintergrund der Gesamttherapiekosten irrelevant.

C BSI erfordern eine sofortige stationäre Aufnahme und intravenöse Antibiotikatherapie.

D BSI können auch durch Translokation aus dem Gastrointestinaltrakt der Patienten verursacht sein.

E BSI reduzieren die Lebensqualität der Patienten und ihrer Familien.

\section{Frage 5}

Welche Aussage zu Präventionsbündeln zum Vermeiden von Gefäßkatheter-assoziierten Infektionen (CA-BSI) bei kinderonkologische Patienten unter einer intensiven Chemotherapie trifft nicht zu?

A Präventionsbündel sind eine vor Ort vereinbarte Zusammenstellung von konkreten Einzelmaßnahmen zur Infektionsprävention.

B In der Kinderonkologie steht dabei die Erhaltungspflege des Broviac- oder Port-Katheters im Vordergrund.

C Präventionsbündel können ohne ausführliche Diskussion im Behandlungsteam aus dem Internet heruntergeladen und übernommen werden.

D Gemeinsam festgelegte Präventionsbündel erleichtern die Schulung und Einarbeitung neuer Mitarbeiter und die Kommunikation mit den Patienten und ihren Angehörigen.

E Es gibt inzwischen Studien aus verschiedenen Ländern, die den Nutzen von Präventionsbündeln in diesem klinischen Kontext belegen.

Weitere Fragen auf der folgenden Seite ... 


\section{Punkte sammeln auf CME. thiene.de}

Fortsetzung ...

\section{Frage 6}

Welche Aussage zu Präventionsbündeln zum Vermeiden von CA-BSI bei kinderonkologischen Patienten unter einer intensiven Chemotherapie ist falsch? Präventionsbündel werden mit höherer Wahrscheinlichkeit vollständig und nachhaltig umgesetzt, wenn...

A sich die entsprechenden Maßnahmen gut in den Arbeitsablauf einpassen (Praktikabilität).

B alle nach einem gemeinsam vereinbarten Standard geschult und trainiert werden.

C genug angemessen qualifiziertes Pflegepersonal zur Verfügung steht.

D alle Patienten mit Chlorhexidin- oder Octenidin-haltigen Waschtüchern behandelt werden.

E eine Überprüfung der Compliance erfolgt.

\section{Frage 7}

Welche Aussage zum Verband der Broviac-Eintrittsstelle ist nicht richtig?

A In Bezug auf das Ziel der Infektionsprävention sind konventionelle (sterile) Pflasterverbände und transparente, semipermeable Folienverbände gleichwertig.

B Bei jedem Verbandswechsel ist eine antiseptische Behandlung der Eintrittsstelle erforderlich.

C Konventionelle Pflasterverbände sollten alle $72 \mathrm{~h}$ gewechselt und täglich palpiert werden.

D Bei granulozytopenischen Patienten können die klassischen Entzündungszeichen bei einer Weichteilinfektion an der Katheter-Eintrittsstelle fehlen oder nur sehr milde ausgeprägt sein.

E Chlorhexidin-freisetzende Verbände sollten grundsätzlich bei allen kinderonkologischen Patienten zum Einsatz kommen.

\section{Frage 8}

Welche Aussage zu Manipulationen an Dreiwegehähnen oder anderen Zuspritzstellen im Infusionssystem trifft nicht zu?

A Bei Verwendung nadelfreier Konnektionsventile ist es ausreichend, diese $5 \mathrm{~s}$ mit einem Reinigungstuch abzuwischen.

B Vor jeder Manipulation soll eine hygienische Händedesinfektion erfolgen.
C Der offene Konus des Dreiwegehahns sollte vor jedem Ansatz einer Spritze/einer Infusionsleitung desinfiziert werden.

D Es ist möglich, den Hub/Dreiwegehahn mit einem geeigneten Antiseptikum einzusprühen, wenn man nach der Einwirkzeit (mind. 15s) die Reste des Antiseptikums auf einer sterilen Kompresse „ausgeklopft“.

E Nach Blutentnahmen oder Bluttransfusionen sind Blutreste am Hub/Dreiwegehahn sehr sorgfältig zu entfernen.

\section{Frage 9}

Welche Aussage zum Wechselintervall des Infusionssystems ist korrekt? Der Systemwechsel sollte ...

A generell immer nach $24 \mathrm{~h}$ erfolgen.

B in der Regel nach $96 \mathrm{~h}$ erfolgen, solange keine lipidhaltigen Infusionslösungen oder Blutprodukte über dieses System verabreicht werden.

C bei Gabe lipidhaltiger Infusionslösungen alle $48 \mathrm{~h}$ erfolgen.

D nur alle 7 Tage erfolgen.

E immer $24 \mathrm{~h}$ nach Gabe eines Erythrozytenkonzentrats erfolgen.

\section{Frage 10}

Welche Aussage zur Rekonstitution/Zubereitung von Arzneimitteln zur i. v. Gabe auf der kinderonkologischen Station trifft zu?

A Eine Ampulle (z. B. mit 400 mg Teicoplanin, „single use only“) darf auf mehrere Patienten aufgeteilt werden.

B Heparin für den Katheterblock sollte aus einer MultidoseHeparin-Ampulle aufgezogen und manuell mit Kochsalz verdünnt werden, weil dies Kosten spart.

C Grundsätzlich muss jede Rekonstitution/Zubereitung von Arzneimitteln zur i.v. Gabe auf der kinderonkologischen Station unter einer Laminar-airflow-Werkbank erfolgen.

D Die planbare Herstellung von komplexen Mischinfusionen zur parenteralen Ernährung soll, wenn möglich, unter Reinraumbedingungen in der Klinikapotheke stattfinden.

E Propofol für die Sedierung (z. B. bei Knochenmarkpunktion) darf morgens um 7:00 Uhr für alle Sedierungspatienten des Vormittags vorbereitet werden. 